\title{
Positive and Negative Sequence Control Strategies to Maximize the Voltage Support in Resistive-Inductive Grids During Grid Faults
}

\author{
Antonio Camacho, Miguel Castilla, Jaume Miret, Member, IEEE, L. García de Vicuña, Ramon Guzman
}

\begin{abstract}
Grid faults are one of the most severe perturbations in power systems. During these extreme disturbances, the reliability of the grid is compromised and the risk of a power outage is increased. To prevent this issue, distributed generation inverters can help the grid by supporting the grid voltages. Voltage support mainly depends on two constraints: the amount of injected current and the grid impedance. This paper proposes a voltage support control scheme that joins these two features. Hence, the control strategy injects the maximum rated current of the inverter. Thus, the inverter takes advantage of the distributed capacities and operates safely during voltage sags. Also, the controller selects the appropriate power references depending on the resistive-inductive grid impedance. Therefore the grid can be better supported since the voltage at the point of common coupling is improved. Several voltage objectives, which cannot be achieved together, are developed and discussed in detail. These objectives are three-fold: a) to maximize the positive sequence voltage, b) to minimize the negative sequence voltage, and c) to maximize the difference between positive and negative sequence voltages. A mathematical optimal solution is obtained for each objective function. This solution is characterized by a safe peak current injection, and by the optimization of the voltage profile in any type of grid connection. Therefore, the proposed control scheme includes advanced features for voltage support during voltage sags, that are applicable to different power facilities in different types of networks. Due to system limitations, a suboptimal solution is also considered, analyzed and discussed for each of the optimization problems. Experimental results are presented to validate the theoretical solutions.
\end{abstract}

Index Terms-Voltage support, Voltage sag, Positive sequence voltage maximization, Negative sequence voltage minimization, Voltage imbalance

\section{INTRODUCTION}

$\mathbf{O}$ $\mathrm{NE}$ of the stringent requirements in grid codes is the voltage ride-through, needed to avoid sudden tripping of power generation due to grid faults. These severe grid perturbations can be the starting point to deteriorate the grid and can led to a blackout if the mitigation activities are not properly implemented. In order to increase the immunity against voltage

This work has been supported by ELAC2014/ESE0034 from the European Union and its linked Spanish national project PCIN-2015-001. We also appreciate the support from the Ministry of Economy and Competitiveness of Spain and European Regional Development Fund (FEDER) under project ENE2015-64087-C2-1-R.

A. Camacho, M. Castilla, J. Miret and L. García de Vicuña. are with the Department of Electronic Engineering, Technical University of Catalonia, 08800 Vilanova i la Geltrú, Spain (e-mail: antonio.camacho.santiago@upc.edu; miquel.castilla@upc.edu; jmiret@eel.upc.edu; vicuna@eel.upc.edu)

R. Guzman is with the Department of Automatic Control, Technical University of Catalonia, 08800 Vilanova i la Geltrú, Spain (e-mail: ramon.guzman@upc.edu) sags, grid codes have evolved from the disconnection to the ride-through, and nowadays to the voltage support strategy. Actual grid codes include some reactive current injection to support the grid voltage so that the risk of disconnection during sags could be minimized to smaller zones close to the fault location. Next generation of grid codes [1]-[3] is being developed to achieve advanced objectives.

These advanced objectives are nowadays possible because power electronics converters are a flexible interface between the generation source and the grid. It is worth mentioning that this flexibility is mainly related to the control implementation. Although the implementation of advanced grid fault control schemes has been improved in recent works for laboratory prototypes, its applicability to real grid conditions is still under development. Nevertheless, most of the control proposals for advanced voltage support during sags are based on symmetric sequences [4]-[16]. The basis of these controllers are the decomposition of the unbalanced grid voltages into the positive and negative sequence voltages. Based on this sequence extraction, the controller computes the power references for positive and negative, active and reactive powers separately. These references are selected based on the control objective and then pass through a stationary reference frame algorithm to build the reference currents. This reference current generator has been widely explained in the literature [10]-[15] and will not be discussed along the work. Instead, the present work focuses on the appropriate selection of the active and reactive, positive and negative power references, so as to optimize the voltage at the terminals of the power inverter. The selection of these power references constitute the main contribution of the work.

It should be noticed that the selection of the voltage support control objective is still an open research topic. Most of the state-of-the-art controllers during grid faults [11]-[14], are applicable for mainly inductive grids, which limits this service to high power systems. However, few works have been proposed for medium and low voltage networks which is one of the main contributions of this work. In [15], a reference generator dealing with the grid impedance is proposed although no voltage objective is formulated. In [16], positive sequence voltage is maximized although only simulation results are reported. The present work extends the results in [16] to a more generic scenario, dealing with positive, negative and both symmetric sequences simultaneously. Also, an optimal and a sub-optimal test case is included to deal with different power capabilities of the inverter. Moreover, experimental results are 
provided to validate the theoretical contributions.

The proposed policies developed along this work are formulated as: a) the maximization of the positive sequence voltage, b) the minimization of the negative sequence voltage, and c) the maximization of the difference between positive and negative sequence voltages. These three objectives have been selected according to different scenarios that include: firstly, the conventional strategy during voltage sags that only injects positive sequence currents. This strategy prioritizes the support of the positive sequence voltage. Secondly, the complementary strategy that tries to minimize as much as possible the voltage imbalance. This strategy is well suited for sags located far from the connection point and for sags with un-faulted phases, since the mitigation of voltage imbalances can help to reduce the risk of disconnection by overvoltage in the phases that do not suffer the sag [17] and improve the transient behavior and immunity against voltage sags for sensitive machines and loads. Finally, the last strategy combines the two previous ones into a more generic and complex problem. This advanced strategy tends to restore the voltage profile as if no sag has occurred, although there exists a physical limitation related to the amount of injected current and the weakness of the grid. All three strategies are formulated for any type of grid impedance, resistive, inductive or a combination of both. Thus, the proposal covers different types of networks.

Each one of the control objectives is developed with the premise that the injected phase currents must be safely controlled to a maximum predefined value. Therefore, the inverter capabilities can be fully exploited because the higher phase current will be safely limited to the maximum rated current of the power converter.

Under some system limitations that will be discussed in detail, the optimal solutions proposed in this paper are unreachable. The limitations for the optimal solutions are related to the amount of active power needed. Although full power inverters are flexible devices to interconnect the power sources and the grid, mainly for reactive power, this flexibility is not yet guaranteed for the active power. This limitation could be overpassed by the proliferation of grid-connected converters with energy storage capacity (such as uninterruptible power sources, or battery-connected static synchronous compensators) that could change quickly the amount of active power needed, or by using the spinning reserves of grid connected power plants. For this reason, each control strategy is being reworked in order to workaround the unfeasible objective. As a result, a suboptimal control policy is proposed and discussed in each optimization problem.

This paper is organized as follows. Section II presents the main concepts involved in the control proposal. Section III, IV and V develop the first, second and third control objectives respectively. Each objective is formulated, the optimal solution is found, the results are analyzed and a suboptimal solution is also proposed, then the experimental results are discussed. Section VI presents the conclusions and future work.

\section{Controller Tools}

This section revisits the basic controller tools that will be needed to develop the proposal. The tools under revision are

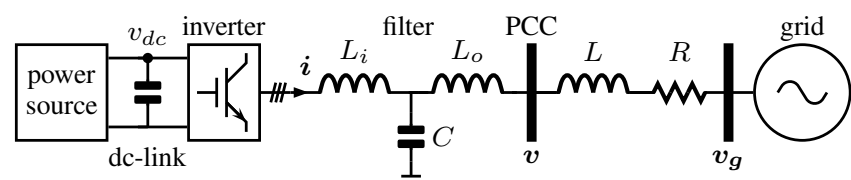

Fig. 1. Simplified scheme of a three-phase grid-connected inverter.

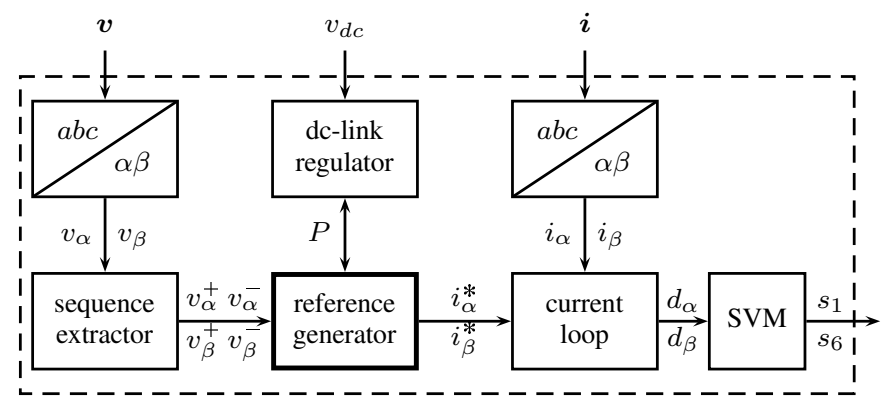

Fig. 2. Control diagram of three-phase inverter under grid fault.

the plant model, the controller architecture, the peak current limitation and the voltage support concept. The overview of the controller parts will help to understand the method to develop the power references that will be the optimal solutions to the above mentioned voltage objectives.

\section{A. Plant Model}

The scheme of a three-phase grid-connecter inverter is shown in Fig. 1. In the scheme, the power source is connected into the grid through a full-power inverter. The inverter uses a $L C L$-filter to reduce noise and switching harmonics. The power plant is connected at the point of common coupling (PCC), which corresponds to the main focus of the proposal, since this is the point where the different strategies must be compared. The grid is modeled by a variable profile grid source that emulates the voltage sag, and an equivalent grid impedance $R$ and $L$. Along this work, it is assumed that the equivalent grid impedance is known. To this end, a basic knowledge of the connection elements close to the PCC must be known, or a method to compute the grid impedance need to be implemented [18]-[22]. In fact, only the inductance/resistance $(L / R)$ ratio is enough for implementing the optimal solutions.

\section{B. Controller Architecture}

The main parts within the voltage support controller during voltage sags are presented in Fig. 2. The controller measures the dc-link voltage $v_{\mathrm{dc}}$, the PCC voltages $\boldsymbol{v}$ and the currents $\boldsymbol{i}$. The dc-link voltage control is needed to maintain a proper active power balance. The grid voltages and currents are transformed into the $\alpha-\beta$ frame. These voltages are decomposed into symmetric sequences. For doing so, a voltage sequence extractor is needed to obtain the sag voltages $v_{\alpha}^{+}$, $v_{\alpha}^{-}, v_{\beta}^{+}$and $v_{\beta}^{-}$at run time. For a complete review about the sequence extraction, see [23] and the references therein. Based on the sequence voltages, the amplitudes of the positive and 
negative sequences are derived. This will serve as a metric for comparing the control proposals

$$
\begin{aligned}
& V^{+}=\sqrt{\left(v_{\alpha}^{+}\right)^{2}+\left(v_{\beta}^{+}\right)^{2}} \\
& V^{-}=\sqrt{\left(v_{\alpha}^{-}\right)^{2}+\left(v_{\beta}^{-}\right)^{2}} .
\end{aligned}
$$

Also, the sag angle $\varphi$ between the positive and negative sequence voltage is needed for developing the proposals. This angle is obtained as

$$
\begin{aligned}
& \cos \varphi=\frac{v_{\alpha}^{+} v_{\alpha}^{-}-v_{\beta}^{+} v_{\beta}^{-}}{V^{+} V^{-}} \\
& \sin \varphi=\frac{v_{\alpha}^{+} v_{\beta}^{-}+v_{\alpha}^{-} v_{\beta}^{+}}{V^{+} V^{-}} \\
& \varphi=\operatorname{atan} 2(\sin \varphi, \cos \varphi)
\end{aligned}
$$

where $\operatorname{atan} 2$ is the two argument arctangent function. Once the voltage sag has been characterized, the reference current generator builds the current references based on the power references and the voltage sequences

$$
\begin{aligned}
& i_{\alpha(\mathrm{p})}^{*}=\frac{2}{3}\left[\frac{v_{\alpha}^{+}}{\left(v_{\alpha}^{+}\right)^{2}+\left(v_{\beta}^{+}\right)^{2}} P^{+}+\frac{v_{\alpha}^{-}}{\left(v_{\alpha}^{-}\right)^{2}+\left(v_{\beta}^{-}\right)^{2}} P^{-}\right] \\
& i_{\beta(\mathrm{p})}^{*}=\frac{2}{3}\left[\frac{v_{\beta}^{+}}{\left(v_{\alpha}^{+}\right)^{2}+\left(v_{\beta}^{+}\right)^{2}} P^{+}+\frac{v_{\beta}^{-}}{\left(v_{\alpha}^{-}\right)^{2}+\left(v_{\beta}^{-}\right)^{2}} P^{-}\right] \\
& i_{\alpha(\mathrm{q})}^{*}=\frac{2}{3}\left[\frac{v_{\beta}^{+}}{\left(v_{\alpha}^{+}\right)^{2}+\left(v_{\beta}^{+}\right)^{2}} Q^{+}+\frac{v_{\beta}^{-}}{\left(v_{\alpha}^{-}\right)^{2}+\left(v_{\beta}^{-}\right)^{2}} Q^{-}\right] \\
& i_{\beta(\mathrm{q})}^{*}=\frac{2}{3}\left[\frac{-v_{\alpha}^{+}}{\left(v_{\alpha}^{+}\right)^{2}+\left(v_{\beta}^{+}\right)^{2}} Q^{+}+\frac{-v_{\alpha}^{-}}{\left(v_{\alpha}^{-}\right)^{2}+\left(v_{\beta}^{-}\right)^{2}} Q^{-}\right]
\end{aligned}
$$

where $P^{+}, P^{-}, Q^{+}$and $Q^{-}$are the power references for positive and negative, active and reactive power respectively. For a detailed discussion about the derivation of these power references see [24] and the references therein. The total current references in the $\alpha-\beta$ channels are obtained by adding the active and reactive components as

$$
\begin{aligned}
& i_{\alpha}^{*}=i_{\alpha(\mathrm{p})}^{*}+i_{\alpha(\mathrm{q})}^{*} \\
& i_{\beta}^{*}=i_{\beta(\mathrm{p})}^{*}+i_{\beta(\mathrm{q})}^{*} .
\end{aligned}
$$

Once the reference currents are obtained, a proportionalresonant current controller compares the references $i_{\alpha}^{*}$ and $i_{\beta}^{*}$ and the measured current values $i_{\alpha}$ and $i_{\beta}$ to obtain the duty cycles $d_{\alpha}$ and $d_{\beta}$. This information passes through a space vector modulator (SVM) to drive the inverter switches $s_{1}-s_{6}$.

The main objective of the proposed work is to find the power references $P^{+}, P^{-}, Q^{+}$and $Q^{-}$that complies two constraints at the same time, maximize a voltage objective function and inject a safely controlled phase current. Thus, the inverter operates in a safe mode and the voltage support is improved because the distributed generation power converter exploits better its own capabilities. Next subsections are focused on the details regarding these objectives.

\section{Peak Current}

The procedure to develop the power references that ensure that the currents will be equal to a maximum rated value $I_{\max }$ is attained based on the inverse Clarke transformation of (6)(9). Assuming that the injected currents follow the references (i.e. $i_{\alpha}^{*} \approx i_{\alpha}$ and $i_{\beta}^{*} \approx i_{\beta}$ ), then the phase current amplitudes can be formulated based on the following relations

$$
\begin{aligned}
& I_{p}^{+}=\frac{2}{3} \frac{P^{+}}{V^{+}} \quad, \quad I_{p}^{-}=\frac{2}{3} \frac{P^{-}}{V^{-}} \\
& I_{q}^{+}=\frac{2}{3} \frac{Q^{+}}{V^{+}} \quad, \quad I_{q}^{-}=\frac{2}{3} \frac{Q^{-}}{V^{-}}
\end{aligned}
$$

where $I_{p}^{+}$and $I_{p}^{-}$are the amplitude of the positive and negative sequences active currents respectively, and $I_{q}^{+}$and $I_{q}^{-}$are the reactive counterparts. Due to the properties of symmetric sequences, positive active and reactive currents are delayed $90^{\circ}$, and the same happens with the negative sequence currents. Joining the positive and negative sequence currents leads to

$$
\begin{aligned}
& I^{+}=\sqrt{\left(I_{p}^{+}\right)^{2}+\left(I_{q}^{+}\right)^{2}} \\
& I^{-}=\sqrt{\left(I_{p}^{-}\right)^{2}+\left(I_{q}^{-}\right)^{2}}
\end{aligned}
$$

where $I^{+}$is the amplitude of the positive sequence active and reactive current, and $I^{-}$is the negative sequence current. Finally, the phase currents amplitudes can be expressed as [24]

$$
\begin{aligned}
& I_{a}=\sqrt{\left(I^{+}\right)^{2}+\left(I^{-}\right)^{2}+2 I^{+} I^{-} \cos \left(\varphi_{I}\right)} \\
& I_{b}=\sqrt{\left(I^{+}\right)^{2}+\left(I^{-}\right)^{2}+2 I^{+} I^{-} \cos \left(\varphi_{I}-2 \pi / 3\right)} \\
& I_{c}=\sqrt{\left(I^{+}\right)^{2}+\left(I^{-}\right)^{2}+2 I^{+} I^{-} \cos \left(\varphi_{I}+2 \pi / 3\right)}
\end{aligned}
$$

where

$$
\varphi_{I}=-\varphi+\operatorname{atan} 2\left(I_{q}^{+}, I_{p}^{+}\right)+\operatorname{atan} 2\left(I_{q}^{-}, I_{p}^{-}\right) .
$$

Eq. (12)-(19) show the relation between the power references $P^{+}, P^{-}, Q^{+}$and $Q^{-}$, and the phase currents $I_{a}, I_{b}$ and $I_{c}$. By conveniently selecting these references, the phase currents can be safely controlled to any predefined value $I_{\max }$.

\section{Voltage Support}

Regarding the voltage support concept, some works have been developed in the literature [13], [25], [26]. The basics for developing the voltage support start from Fig. 1 where the instantaneous PCC voltages can be expressed as

$$
\begin{aligned}
& v_{\alpha}=v_{g \alpha}+R i_{\alpha}+L \frac{d i_{\alpha}}{d t} \\
& v_{\beta}=v_{g \beta}+R i_{\beta}+L \frac{d i_{\beta}}{d t}
\end{aligned}
$$

where $v_{\alpha}$ and $v_{\beta}$ are the voltages at the PCC in the $\alpha$ - $\beta$ channels, $v_{g \alpha}$ and $v_{g \beta}$ are the sag voltages at the grid side, and $R$ and $L$ are the resistive and inductive grid impedance. By the decomposition of the voltages and currents in (20) and (21) into the symmetric components, and by using (6)-(9), the amplitudes of the involved magnitudes can be derived. The following relations hold for the amplitudes of the positive and negative sequence at the PCC side

$$
\begin{aligned}
& V^{+}=R I_{p}^{+}+\omega L I_{q}^{+}+\sqrt{\left(V_{g}^{+}\right)^{2}-\left(\omega L I_{p}^{+}-R I_{q}^{+}\right)^{2}} \\
& V^{-}=R I_{p}^{-}-\omega L I_{q}^{-}+\sqrt{\left(V_{g}^{-}\right)^{2}-\left(\omega L I_{p}^{-}+R I_{q}^{-}\right)^{2}} .
\end{aligned}
$$


The main idea of this paper can be understood from (22) and (23). Assuming some constant value for $V_{g}^{+}$, which strategy will be preferred to raise as much as possible $V^{+}$, or equivalently, what the references for positive active and reactive currents should be, provided that they must be kept within a limited safety value. Similarly one can develop the negative references to minimize the negative sequence voltage. Finally the complex problem arises when dealing with both sequences simultaneously. These three objectives will be developed separately within Sections III, IV and V.

From these equations, it can be concluded that the optimal solution is closely linked to the equivalent grid impedance. Therefore, a well known grid model or an on-line method to estimate the impedance value is required [18]-[22]. In case of a wrong grid estimation, then the solution will not be optimal. Even so, the solution will help to support the grid voltage and to exploit the inverter capabilities by injecting the maximum rated current during the sag.

To sum up, the control proposal formulates three different objective functions related to $V^{+}, V^{-}$and the difference $V^{+}-$ $V^{-}$which depend on the variables $P^{+}, P^{-}, Q^{+}$and $Q^{-}$, the plant parameters $R$, and $L$ and the safety restriction for the maximum admissible current $I_{\max }$.

\section{Strategy A: Maximization of the Positive SEQUENCE Voltage}

The first strategy proposed in this work maximizes the positive sequence voltage at the PCC by appropriately selecting the positive active and reactive powers. This strategy has some inherent benefits since only positive sequence powers are injected.

\section{A. Problem Formulation}

The problem relays in the mathematical computation of the positive sequence active and reactive powers that comply simultaneously the two control premises:

- safely inject the maximum rated current of the inverter,

- maximize the positive sequence voltage at the PCC.

The objective of the control strategy is formulated as

$$
\max V^{+}\left(P^{+}, Q^{+}\right)
$$

subject to: $\max \left\{I_{a}, I_{b}, I_{c}\right\}=I_{\max }$.

\section{B. Optimal Solution}

The optimal solution is found by using Lagrange multipliers. The problem consists in building the Lagrange function $\mathcal{L}$ based on the objective function $f$ and the restriction $g$ so that

$$
\mathcal{L}(x, y, \lambda)=f(x, y)+\lambda g(x, y)
$$

where the objective function is derived by substituing (12)-(13) in $(22)$,

$$
f(x, y)=V^{+}\left(P^{+}, Q^{+}\right)
$$

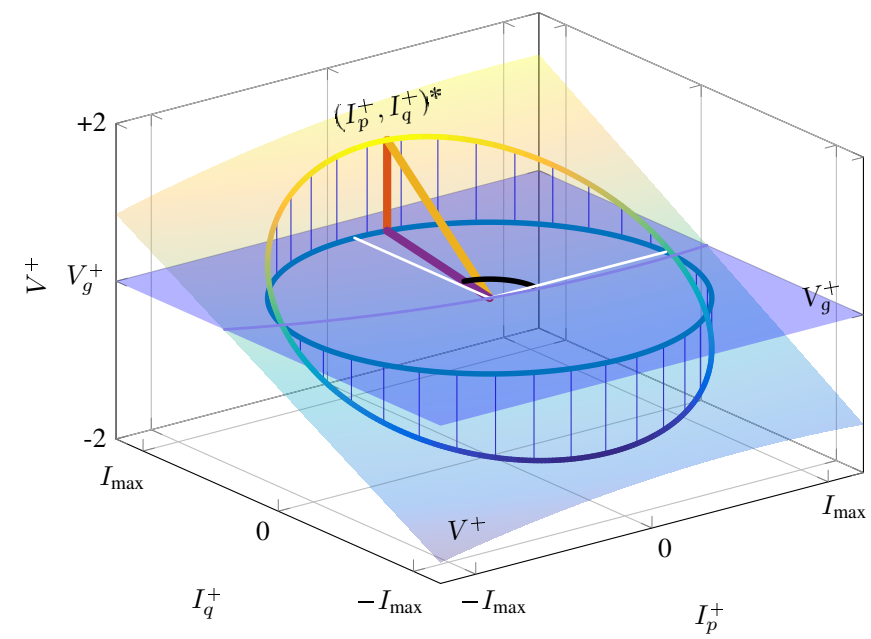

Fig. 3. Graphical solution for the maximization of the positive sequence voltage.

$\lambda$ is the Lagrange multiplier, and the restriction is arranged based on (12)-(13) taking into account that $I_{p}^{-}=I_{q}^{-}=0$.

$$
g(x, y)=\sqrt{\left(\frac{2}{3} \frac{P^{+}}{V^{+}}\right)^{2}+\left(\frac{2}{3} \frac{Q^{+}}{V^{+}}\right)^{2}}-I_{\max } .
$$

Once the Lagrange multiplier is formulated, the gradient with respect to the involved variables $P^{+}, Q^{+}$and $\lambda$ is obtained. Then the solution is obtained equaling the gradient to 0

$$
\nabla \mathcal{L}(x, y, \lambda)=\left(\frac{\partial \mathcal{L}}{\partial P^{+}}, \frac{\partial \mathcal{L}}{\partial Q^{+}}, \frac{\partial \mathcal{L}}{\partial \lambda}\right)=0 .
$$

Solving (28), the pair of points $(x, y)^{*}$ that represent the optimal solution to (24) is

$$
\begin{aligned}
& \left(P^{+}\right)^{*}=\frac{3}{2} \frac{R}{\sqrt{R^{2}+(\omega L)^{2}}} I_{\max } V^{+} \\
& \left(Q^{+}\right)^{*}=\frac{3}{2} \frac{\omega L}{\sqrt{R^{2}+(\omega L)^{2}}} I_{\max } V^{+} .
\end{aligned}
$$

The solution to the problem indicates that there exist an optimal point $\left(P^{+}, Q^{+}\right)^{*}$ where both objectives in (24) are accomplished: the positive sequence voltage is maximized and the injected currents equal the rated current of the inverter. This point depends on the impedance values $R$ and $L$ as expected. The above solution represents the optimal solution for any grid impedance. Therefore, the voltage support can be improved by using these references. Following the analysis of these expressions, two extreme cases should be discussed. It is clear that the solution for purely inductive grids is $I_{p}^{+}=0$ and $I_{q}^{+}=I_{\max }$, or equivalently $P^{+}=0$ and $Q^{+}=3 / 2 I_{\max } V^{+}$. In the other extreme case for purely resistive grids, the complementary solution to optimize the voltage support is $I_{p}^{+}=I_{\max }$ and $I_{q}^{+}=0$, or $P^{+}=3 / 2 I_{\max } V^{+}$and $Q^{+}=0$.

The solution in (29) and (30) is graphically presented in Fig. 3. In order to better analyze the graph, the axes of the plot are the active current $I_{p}^{+}$and the reactive one $I_{q}^{+}$ instead of the power references $P^{+}$and $Q^{+}$. In the z-axis, the resulting voltage support for these variables is shown. Two 
planes are drawn, the horizontal one representing a constant value $V_{g}^{+}$for the perturbed grid voltage, and the tilted one that shows the voltage support effects as the value for $V^{+}$in different current combinations $I_{p}^{+}$and $I_{q}^{+}$. The peak current limitation is shown as a circle when projected over the $I_{p}^{+}-I_{q}^{+}$ plane, and as an ellipse over the $V^{+}$surface. These values correspond to the points that comply with the $I_{\max }$ restriction in (27). Among all the possible solutions, the higher point is marked as the optimal solution $\left(I_{p}^{+}, I_{q}^{+}\right)^{*}$, which represents the local maximum of the objective function subject to the current constraint. The figure has been composed based on some arbitrary plant parameters $\omega L>R$. For other types of grid impedance, the voltage support effects will change, depending on the stiffness or weakness of the grid, and the $L / R$ ratio.

This figure clearly shows the contributions of this paper. The voltage support optimization depends on the equivalent grid impedance. Therefore, the proposed advanced controller can help to better support the grid voltage by taking into account the $L / R$ ratio. By maximizing the voltage support, the voltage ride through is improved and the disconnection of the power facility can be reduced to smaller regions close to the location where the grid fault occurs. Whenever the grid impedance is wrongly estimated, the solution is no longer optimal. However, even in this case, the solution will be helpful in terms of voltage support since the inverter will inject its maximum available current and the balance between active and reactive power references will feed and support the grid simultaneously.

\section{Sub-optimal Solution}

A close inspection to (29) shows that the optimal active power injection $\mathrm{P}^{+}$could be less than the generated power. Therefore, active power curtailment strategies need to be implemented to accomplish with the proposed optimal solution for the maximization of the positive sequence voltage. Active crowbars, detuning controllers or battery storage systems can be needed to achieve this objective. Similarly, (29) shows that the optimal solution can lead to an unreachable active power reference $P^{+}$. This issue can be due to a low production scenario where the active power production is lower than the optimal requirement. In such a case, the optimal solution can not be implemented without spinning reserves [27] or energy storage elements that can supply the extra power needed. In such a case, then the proposed references need to be modified accordingly by limiting the injected active power and increasing the reactive one till the maximum rated current of the inverter is reached. Therefore the sub-optimal solution is a workaround for the unreachable optimal solution that can be suited for the operation of any power converter in any situation. Thus, improving the voltage support under any circumstance.

In order to deal with the unfeasible active power production, the optimal solutions (29) and (30) when an arbitrary active power $P$ is injected or when the active power reference can
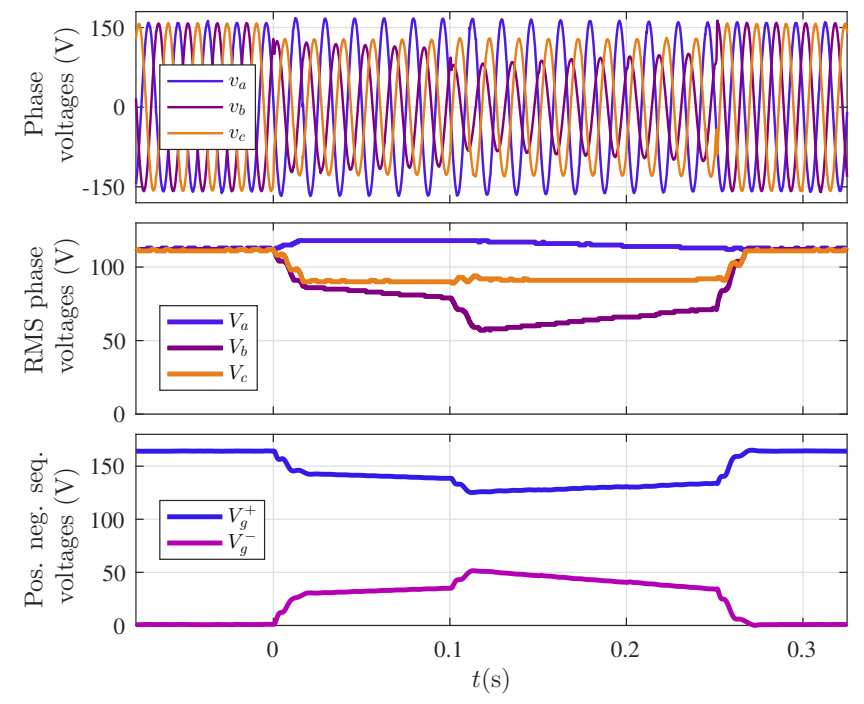

Fig. 4. Sag characterization for the experimental scenario.

not be reached are modified to be

$$
\begin{aligned}
\left(P^{+}\right)^{\star} & =P \\
\left(Q^{+}\right)^{\star} & =\sqrt{\left(\frac{3}{2} I_{\max } V^{+}\right)^{2}-P^{2}} .
\end{aligned}
$$

The solution, although is not optimal in terms of maximizing the positive sequence voltage, improves the behavior of the inverter during grid faults by safely injecting the maximum rated current. Note that the grid impedance should not be known in this case.

\section{Experimental Results}

In order to validate the proposed control scheme, a laboratory prototype has been built according to Fig. 1. The experimental setup is composed by an Amrel DC power source, a Guasch three phase inverter and a Pacific AC power source to get repetitive voltage sags. A complete description of the setup is included at the end of the paper, in the Appendix.

The system parameters are collected in Table I. The controller is implemented on a F28M36 Texas Instruments digital signal processor. The main parts of the control algorithm are the second order generalized integrators [28] used to extract the voltage sequences at run-time, and the reference generator that set the reference powers $P^{+}, P^{-}, Q^{+}$and $Q^{-}$since this is the main contribution of the paper and the kernel for the implementation of the proposal. Also, a proportional-resonant controller and a SVM are implemented in the current loop. To highlight the voltage support effects, a weak grid is considered with impedance values $R=0.06$ p.u. and $L=0.12$ p.u.

A complex sag has been programmed in the grid emulator to analyze the behavior of the proposed control scheme. This voltage sag will be used along all the experiments to test the performance of the proposal under the same grid fault. The sag is presented in Fig. 4. The top of the figure shows the instantaneous phase voltages. The middle of the plot presents the root mean square (RMS) voltage values. And the bottom part is related to the positive $V_{g}^{+}$and negative $V_{g}^{-}$sequence 


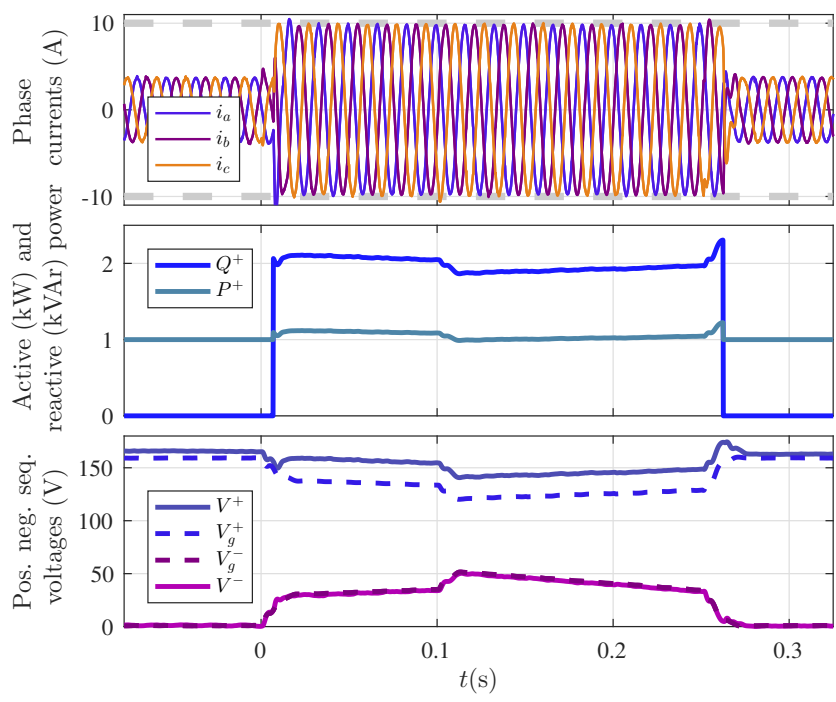

Fig. 5. Experimental results for Strategy A. Phase currents, power references and sequence voltages.

voltages at the grid side, therefore this will be the graph where the objective functions are compared. The sag starts at $t=0$, and has two transition segments to better emulate complex grid faults. Once the sag has been detected, the control proposal is launched and the results are discussed below. The sag detector is based on the comparison of the rms phase voltages with a threshold of 0.80 p.u. Below this threshold, the grid is considered in fault-mode. This detection method helps to fastly react to the perturbance. Similarly, the fault is cleared whenever the three-phase rms voltages are above 0.85 p.u.

The experimental results for the problem stated in (24) are presented in Fig. 5. Before the sag, the inverter operates in normal mode and set the active power to $P=1000 \mathrm{~W}$, and $Q=0$ VAr. Upon the sag detection, the controller computes the reference powers $P^{+}$and $Q^{+}$according to (29) and (30). Once the sag is cleared, the controller turns back to the normal operation mode. The first objective is to take advantage of the maximum rated current of the inverter when supporting the grid voltage during the fault $\left(I_{\max }=10 \mathrm{~A}\right.$ along the experiments). As it can be seen in the top of the figure, the peak value of the injected currents during the sag is $I_{\max }$, therefore the first objective of the control proposal is accomplished. The second objective is to maximize the positive sequence voltage $V^{+}$, as can be seen in the bottom of the figure, where the amplitudes of the positive and negative sequences at the grid and at the PCC side are compared. According to these optimal solutions, this is the maximum positive sequence voltage that can be reached at the PCC taking into account the current limitation $I_{\max }$. Other strategies will result in a lower value for $V^{+}$, or in a tripping of the inverter by overcurrent. To achieve the above objectives, the middle part of the figure shows the power references that are being computed during the sag according to (29) and (30). It should be mentioned that these references evolve according to the dynamic voltage measured at the PCC side for the programmed voltage sag.

Once the optimal solution has been presented, a discussion

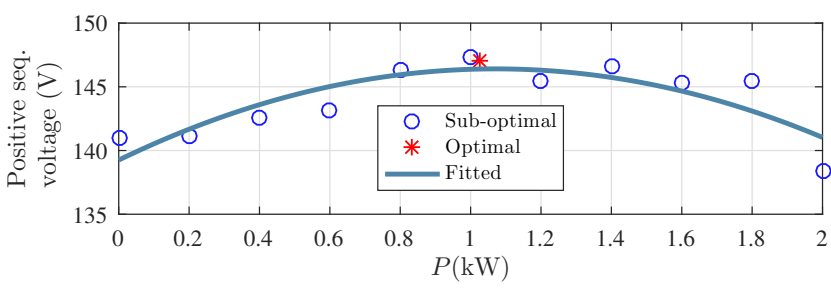

Fig. 6. Sub-optimal results for Strategy A. Voltage support for different active power at time $t=0.2 \mathrm{~s}$.

should be included to compare the optimal and sub-optimal solutions. To this end, a collection of results have been experimentally obtained. The results are presented in Fig. 6. The values plotted correspond to a snapshot of the voltage support effects gathered at time $t=0.2 \mathrm{~s}$ (this value in chosen arbitrarily inside the sag interval). The tests are performed by selecting different values of the active power reference $P$ in (31) and (32).

The red mark corresponds to the optimal solution, while the blue ones belong to the suboptimal values. Also a fitting curve is included in the graph. These results show that the maximum positive voltage increment is obtained at the optimal point computed as in (29)-(30), while less voltage support is obtained when the active power reference moves away from this point. The reason for these results rely on the discrepancy between the optimal and sub-optimal power references, as could be seen from Fig. 3 when the references move away from the optimal solution, or from (22) where it can be understood the different voltage support obtained when injecting different active and reactive powers, where only one of the solutions is optimal.

Comparing the experimental results for the proposed control strategy and other control schemes presented in the literature, the proposed solution has the capability to increase the positive sequence voltage more than any other solution whenever the grid has some inductive and resistive behaviour. For instance, a $16 \%$ increment of the positive sequence voltage is obained with the proposal compared to the $12 \%$ increment reached with the balanced control strategy reported in [29] where only reactive power is used to support the grid (note that a weak grid is considered). This $33 \%$ relative increment can help to better ride-through severe grid faults, and to support the grid in a distributed manner. This is accomplished by taking into account the grid impedance, and by limiting the maximum rated current of the inverter, which is of interest during faults. The peak current capability is attained with low computational overhead, compared with [24]. As a drawback, it is worth mentioning that the instantaneous powers will exhibit oscillations at twice the grid voltage due to the imbalance in the system, with a maximum peak value of $700 \mathrm{~W}$ for the worst case in the sag under test. However, the average value of these oscillations coincide with the power references thanks to the use of the reference generator in (6)-(9). It should be noted that these oscillations could affect the performance of the grid-connected inverter. 


\section{Strategy B: Minimization of the Negative SEQUence Voltage}

Next strategy is intended to minimize the negative sequence voltage $V^{-}$which appears whenever a one or two phase voltage sag occurs. The minimization of negative sequence voltage during grid faults, which is the focus of this study, has some interesting benefits specially for the un-faulted phase(s) [17], [25] and for sags located far from the facility, since power quality and machine operation quickly deteriorates as the imbalance grows.

\section{A. Problem Formulation}

For this second control strategy, the problem can be formulated as

$$
\begin{aligned}
\min & V^{-}\left(P^{-}, Q^{-}\right) \\
\text {subject to: } & \max \left\{I_{a}, I_{b}, I_{c}\right\}=I_{\max }
\end{aligned}
$$

where the objective function is to reduce as much as possible the negative sequence voltage at the PCC subject to the injection of a current that will be fixed to a desired safety value.

\section{B. Optimal Solution}

The same procedure as presented in previous strategy is adopted here. The solution of the problem is obtained via Lagrange multipliers to get the critical points. Firstly the gradient with respect to the involved variables and the Lagrange multiplier is obtained. Once the gradient is set to zero and solved for the involved variables, the solution to (33) is obtained

$$
\begin{aligned}
& \left(P^{-}\right)^{*}=\frac{-3}{2} \frac{R}{\sqrt{R^{2}+(\omega L)^{2}}} I_{\max } V^{-} \\
& \left(Q^{-}\right)^{*}=\frac{3}{2} \frac{\omega L}{\sqrt{R^{2}+(\omega L)^{2}}} I_{\max } V^{-} .
\end{aligned}
$$

From (34) and (35), it is clearly shown that the optimal solution depends on the grid impedance as expected. Also, an important remark should be highlighted regarding the sign of the active power, which should be negative in any case. Therefore, this strategy has some particularities that need to be discussed in detail. The main issue when implementing this optimal solution is the capability of a power system to absorb active power. This is only possible when a backup system for energy storage is connected to the generation system, or when a dissipative dc-link voltage controller is implemented to burn the excess of energy.

As in the previous case, a graphical interpretation for the optimal policy when minimizing the negative sequence voltage is presented in Fig. 7. In this case, the optimal solution is below the $V_{g}^{-}$plane that represents the negative sequence voltage at the grid side, because the effect of supporting the grid voltages is to decrease the negative sequence voltage at the PCC side. The figure has been plotted with $\omega L>R$, and the optimal solution for the reactive current $\left(I_{q}^{-}\right)^{*}$ is close to $I_{\max }$. Also, this plot clearly shows the problem with the

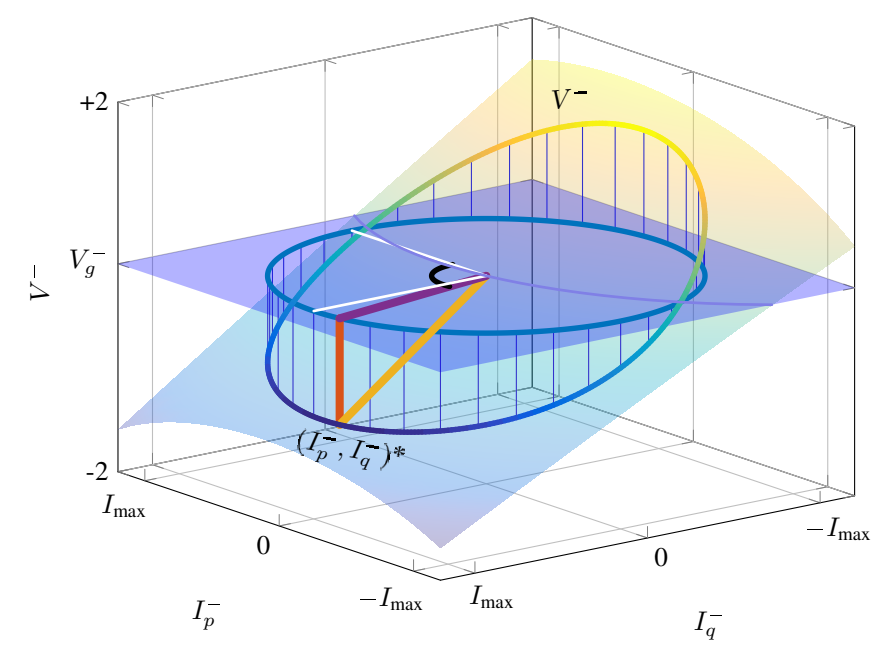

Fig. 7. Graphical solution for the minimization of the negative sequence voltage.

negative values for the active current $I_{p}^{-}<0$ respresenting a negative value for the active power reference.

\section{Sub-optimal Solution}

To workaround the negative active power limitation, the optimal solution presented in (34) and (35) is arranged to the suboptimal case. For those systems that cannot implement negative values for the active power reference, the sub-optimal strategy is to set $P^{-}=0$. Thus, all the injected current will be reactive.

$$
\begin{aligned}
\left(P^{-}\right)^{\star} & =0 \\
\left(Q^{-}\right)^{\star} & =\frac{3}{2} I_{\max } V^{-} .
\end{aligned}
$$

This solution guarantees a sub-optimal solution without the above-mentioned drawback.

\section{Experimental Results}

The experimental results for strategy B have been tested against the same voltage sag as in the previous case. See Fig. 4 for the sag characterization and the Appendix for a complete experimental description. The experimental results for the second strategy are shown in Fig. 8. The figure includes the injected phase currents in the top, the power references in the middle and the voltage support effect in the bottom. As a constraint added in the case studies, it can be seen in the upper part of the figure that the phase currents are perfectly controlled to the peak current value $I_{\max }=10 \mathrm{~A}$, in order to fully exploit the inverter capacities. The power references during the sag, corresponding to the optimal solution in (34)(35) are shown in the middle. As it can be appreciated, $P^{-}$ and $Q^{-}$evolves as the sag does in order to fix the rated current and minimize $V^{-}$. Finally, the negative sequence voltage at the PCC $V^{-}$is reduced when compared with the grid one $V_{g}^{-}$, as shown below in the graph. Contrary to the previous test, it should be mentioned that this strategy only affects the negative sequence voltage while the positive one remains unsupported along the sag. This strategy is the best in terms of reducing 


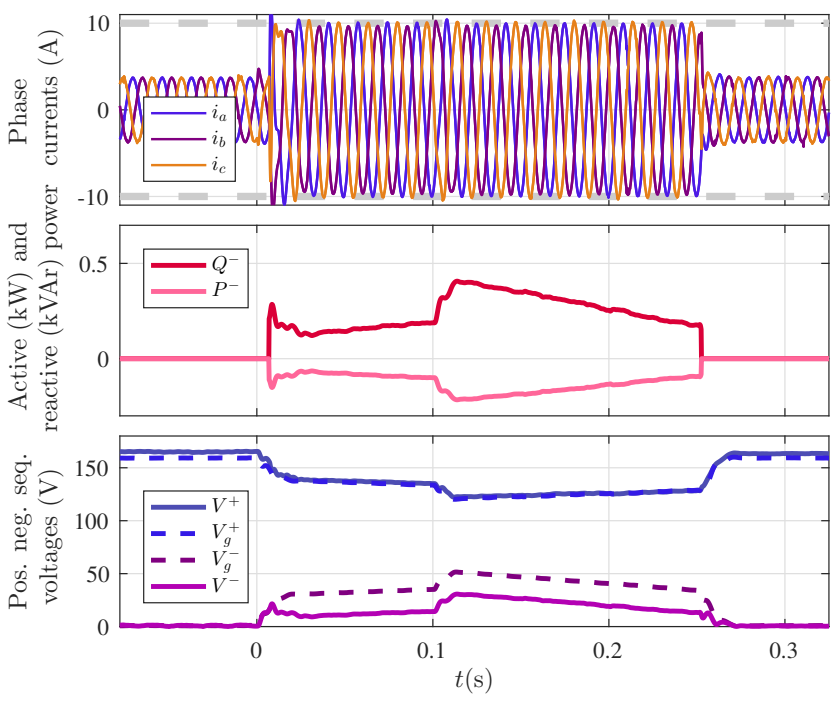

Fig. 8. Experimental results for Strategy B. Phase currents, power references and sequence voltages.

the negative sequence voltage taking into account the current limitation as it can be seen from the bottom of the figure.

To further discuss the solution, the suboptimal case is also compared. As opposed to previous strategy A, the sub-optimal solution (36) and (37) for the minimization of the negative sequence voltage is unique (i.e. $P=0$ ). Therefore, only one comparison point is discussed corresponding to the voltage support at $t=0.2 \mathrm{~s}$ for the optimal and sub-optimal solutions. For the optimal case $V^{-}$has been reduced from $37.7 \mathrm{~V}$ to 18.3V. As expected, for the sub-optimal solution, less voltage reduction is produced, from $37.7 \mathrm{~V}$ to $20.4 \mathrm{~V}$. Therefore, the optimal solution improves a $10.8 \%$ the reduction of the negative sequence voltage compared with the sub-optimal solution.

Comparing this strategy with other possible policies, the proposed one minimizes the negative sequence voltage. Therefore, no other strategy performs better when dealing with this objective. As a drawback, the proposed strategy cannot deal with positive sequence voltage. To improve this behavior under any type of grid fault, next strategy is focused on both symmetric sequences simultaneously.

\section{Strategy C: Maximization of the Difference} Between Positive and Negative Sequence Voltages

Strategy A deals with the maximization of the positive sequence voltage, while strategy $B$ is related to the minimization of the negative sequence. The next strategy combines these two problem formulations into a generic optimization problem. The objective for the strategy $\mathrm{C}$ is to increase as much as possible the distance between the positive and the negative sequence voltages. From a system point of view, this strategy has some noticeable advantages, as for example the combination of positive and negative sequence powers to support both sequences. Also the optimal solution has some active and some reactive components which is helpful when simultaneously feeding and supporting the grid. This strategy makes use of the whole capacities of the inverter by injecting the rated current, and improves the voltage support as no other strategy can perform. The outstanding results for this strategy come from the fact that the best strategy during grid faults is a complex decision that involves many variables. In terms of immunity, this strategy tends to move the voltages closer to the case when no fault occurs subject to the limitation of the maximum current injection and the grid weakness.

\section{A. Problem Formulation}

The problem can be formulated as

$$
\max V^{+}\left(P^{+}, Q^{+}\right)-V^{-}\left(P^{-}, Q^{-}\right)
$$$$
\text { subject to: } \quad \max \left\{I_{a}, I_{b}, I_{c}\right\}=I_{\max } \text {. }
$$

\section{B. Optimal Solution}

The solution to (38) is a 4-tuple representing the variables for the power references $P^{+}, P^{-}, Q^{+}$and $Q^{-}$. The optimal solution to improve the voltage support is presented below

$$
\begin{aligned}
& \left(P^{+}\right)^{*}=\frac{3}{2} \frac{I_{\max }}{\sqrt{2}} \frac{V^{+}}{\sqrt{3}} \frac{R+R \cos \hat{\varphi}-\omega L \sin \hat{\varphi}}{z \sqrt{R^{2}+(\omega L)^{2}}} \\
& \left(P^{-}\right)^{*}=\frac{-3}{2} \frac{I_{\max }}{\sqrt{2}} \frac{V^{-}}{\sqrt{3}} \frac{R+R \cos \hat{\varphi}+\omega L \sin \hat{\varphi}}{z \sqrt{R^{2}+(\omega L)^{2}}} \\
& \left(Q^{+}\right)^{*}=\frac{3}{2} \frac{I_{\max }}{\sqrt{2}} \frac{V^{+}}{\sqrt{3}} \frac{\omega L+\omega L \cos \hat{\varphi}+R \sin \hat{\varphi}}{z \sqrt{R^{2}+(\omega L)^{2}}} \\
& \left(Q^{-}\right)^{*}=\frac{3}{2} \frac{I_{\max }}{\sqrt{2}} \frac{V^{-}}{\sqrt{3}} \frac{\omega L+\omega L \cos \hat{\varphi}-R \sin \hat{\varphi}}{z \sqrt{R^{2}+(\omega L)^{2}}} .
\end{aligned}
$$

where

$$
z=\max \{\sqrt{1+\cos (\hat{\varphi})}, \sqrt{1+\cos (\hat{\varphi}-2 \pi / 3)}, \sqrt{1+\cos (\hat{\varphi}+2 \pi / 3)}\}
$$

and

$$
\hat{\varphi}:=\left\{\begin{array}{rlrl}
\varphi, & -60^{\circ} & \leqslant \varphi<60^{\circ} \\
\varphi-2 \pi / 3, & 60^{\circ} \leqslant \varphi<180^{\circ} \\
\varphi+2 \pi / 3, & 180^{\circ} \leqslant \varphi<-60^{\circ}
\end{array}\right.
$$

In order to ensure a safely current injection, (43) and (44) are needed to implement the proposal during any type of voltage sag.

The above expressions constitute the major contribution of this paper. With them, the voltage support is optimized for any grid impedance combination ( $R$ and $L$ ) and voltage sag profile $\left(V^{+}, V^{-}\right.$and $\left.\varphi\right)$. Note that, in strategy $\mathrm{C}$, the power references depend on the sag angle between positive and negative sequence voltage, as opposed to strategy A and B. Therefore, the on-line measurement of this angle is fundamental in this strategy since it determines the contribution of the resistive and inductive grid impedances in the power references.

A close inspection of (39)-(44) reveals some interesting properties. The following relations are derived from the optimal solution.

$$
\begin{aligned}
& I^{+}=I^{-} \\
& \varphi_{I}=\pi .
\end{aligned}
$$


These relations imply that the solution for the optimization problem proposed in (38) is obtained when the amplitude of the positive and negative sequence currents coincide. Moreover, the angle between the sequence currents and the sequence voltages is equal to $180^{\circ}$.

\section{Sub-optimal Solution}

As in the case of strategies A and B, the optimal solution could not be feasible due to active power limitations. To workaround this issue, a sub-optimal strategy for this case is also proposed. The basis for the sub-optimal case should contain positive and negative sequence powers so as to mimic the optimal solution. However, to avoid the restrictions in the active power, $P$ has been selected to null during the sag. Thus, during the sag all the injected powers will be reactive. Also, the balance between positive and negative reactive power is selected so as to produce a voltage increase in the positive sequence voltage which should be similar to the decrease in the negative sequence voltage (i.e. $\Delta V^{+} \approx \Delta V^{-}$). To this end, the following simplification from (22) and (23), which is certain for purely inductive grids, is taken into account

$$
\begin{aligned}
& \Delta V^{+}=V^{+}-V_{g}^{+} \approx \omega L I_{q}^{+}=\omega L \frac{2 Q^{+}}{3 V^{+}} \\
& \Delta V^{-}=V_{g}^{-}-V^{-} \approx \omega L I_{q}^{-}=\omega L \frac{2 Q^{-}}{3 V^{-}} .
\end{aligned}
$$

In order to ensure $\Delta V^{+} \approx \Delta V^{-}$, from (47) and (48), the following holds

$$
\frac{Q^{+}}{V^{+}}=\frac{Q^{-}}{V^{-}}
$$

Taking into account that $P=0$, and the relation in (49), the sub-optimal solutions for a safe operation of the inverter are

$$
\begin{aligned}
& \left(P^{+}\right)^{\star}=0 \\
& \left(P^{-}\right)^{\star}=0 \\
& \left(Q^{+}\right)^{\star}=\frac{3}{2} \frac{I_{\max }}{\sqrt{2}} \frac{V^{+}}{z^{\prime}} \\
& \left(Q^{-}\right)^{\star}=\frac{3}{2} \frac{I_{\max }}{\sqrt{2}} \frac{V^{-}}{z^{\prime}}
\end{aligned}
$$

where

$$
z^{\prime}=\max \{\sqrt{1-\cos (\hat{\varphi})}, \sqrt{1-\cos (\hat{\varphi}-2 \pi / 3)}, \sqrt{1-\cos (\hat{\varphi}+2 \pi / 3)}\}
$$

and $\hat{\varphi}$ is defined as in (44).

The sub-optimal solution in (50)-(54) presents a simplified method to support both sequences with reactive power. The combination of positive and negative powers inherently imposes a current imbalance. To avoid the overcurrent tripping, (54) ensures that the maximum injected current will be $\max \left\{I_{a}, I_{b}, I_{c}\right\}=I_{\max }$.

\section{Experimental Results}

Fig. 9 shows the experimental results for the optimal solution presented in (39)-(42). The same voltage sag as in the previous experiments is also tested during this strategy.

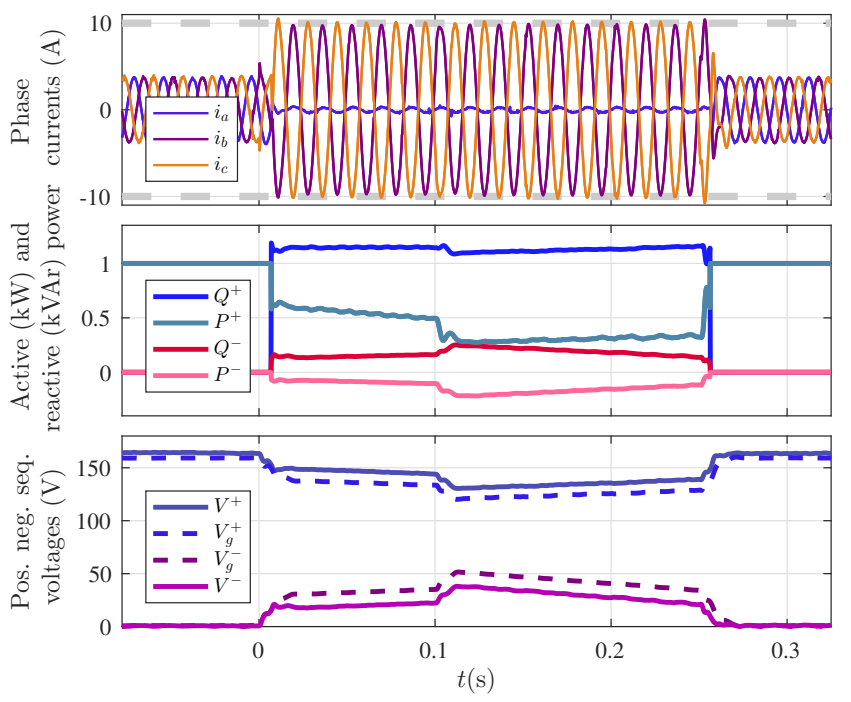

Fig. 9. Experimental results for Strategy C. Phase currents, power references and sequence voltages.

The upper plot presents the phase currents, where it can be seen that the currents are unbalanced because the injection of positive and negative sequence currents yields to different amplitudes in each phase. However, the main objective of safely inject a maximum current limited to the predefined value $I_{\max }$ is still guaranteed. The middle plot shows the power references where the time evolution of the optimal solution is shown. It should be noted that each power component has a contribution on the voltage support so as to maximize the objective function. This contribution is shown in the bottom part of the figure, where it can be appreciated that both the positive and the negative sequences voltages are supported. Therefore the objective function $V^{+}-V^{-}$is maximized. It is worth mentioning that the proposed strategy tends to support the phase voltages at the PCC to the pre-fault voltages (normal operation), since the positive sequence voltage tends to be increased and the negative one tends to be removed from the system.

After presenting the optimal solution for this strategy, the sub-optimal one is also validated. For the optimal case, the positive sequence voltage variation at $t=0.2 \mathrm{~s}$ is $\Delta V^{+}=$ $9.9 \mathrm{~V}$, and the negative one is $\Delta V^{-}=13.2 \mathrm{~V}$. However, for the sub-optimal case, the results are $\Delta V^{+}=9.7 \mathrm{~V}$ and $\Delta V^{-}=9.6 \mathrm{~V}$. As a result, the objective function to be maximized $V^{+}-V^{-}$is $108.1 \mathrm{~V}$ in the optimal policy and a a bit lower $105.3 \mathrm{~V}$ for the sub-optimal strategy. The main reason for this difference is that (39)-(42), and the relations stated in (45) and (46) ensure a optimal leverage of the injected currents for any $R / L$ grid impedances, while (50)-(53) are unable to achieve the same results.

As shown along the experiments, this last strategy presents some advances with other voltage support strategies. The most important is that both sequences, positive and negative, are supported. The simultaneous voltage support could be a promising alternative to help the grid during the fault, combining the benefits of both solutions into one single policy. 


\section{CONClusions AND Future Work}

This paper has presented a voltage support control strategy during grid faults with two objectives: inject the maximum rated current of the inverter and maximize a voltage support objective function. The achieved objectives enhance the ride through protocols for voltage sags because can make a better utilization of the distributed capacities of grid-connected inverters. The experimental results have demonstrated the applicability of the proposal even in complex sags with a timevarying voltage profile.

Among the inherent benefits of the control proposals, three main aspects should be highlighted: i) the inverter operates safely during the grid fault, ii) the whole current capacity is used to support the grid voltage, and iii) the voltage support is optimized for different grid impedances. The proposed strategies present a wide range of applications, depending on what is preferred during the sag, either increase the positive sequence voltage, or decrease the negative sequence, or a combination of both. Therefore the control policies can be flexibly implemented according to the needs of the power system where the power source is located.

The mathematical solution to the optimization problems ensures that the proposed strategies can help the grid to avoid the unwanted trip-off effects, and therefore contribute to increase the grid reliability during this kind of extreme perturbations, preventing the risk of a grid outage in a distributed manner. It should be noticed that this control scheme includes, but it is not limited to high power facilities, since smaller power sources or any inverter-based device with flexible operation can help to support the grid voltage with the proposed strategy even in weak or stiff, resistive or inductive grids.

Although the proposed strategies depend on the impedance estimation, it should be noticed that a perfect knowledge of the $L$ and $R$ values is not really needed, since the strategies produce small voltage differences in a wide range around the optimal impedance. Therefore, the proposed strategies can help to support the grid even when some uncertainty exists between the real and the estimated impedances.

Future work will be focused on the selection of the appropriate objective function under different scenarios, and the smart implementation of the optimal or sub-optimal strategy.

\section{APPENDIX}

This appendix deals with the experimental setup used for testing the proposed control strategies. The test platform consists on a laboratory prototype based on the scheme presented in Fig.1. The main parts of the system are a 6kVA Pacific Power AC voltage source, a 5kW Amrel DC power source and a $2.3 \mathrm{kVA}$ Guasch three-phase inverter. The system parameters are listed in Table I. Custom hardware has been developed for sensing the inverter voltages and currents, and driving the inverter's switches.

The controller has been implemented on a F28M36 control card from Texas Instruments. The code is fully written in Clanguage. The firmware being programmed into the controller can be divided into two different parts: supervision and control. For supervision, several configurable buffers are capable
TABLE I

SYSTEM PARAMETERS

\begin{tabular}{lccc}
\hline \hline & Symbol & Nominal value & p.u. value \\
\hline base power & $S_{e}$ & $2.3 \mathrm{kVA}$ & 1 \\
rated current & $I_{\max }$ & $10 \mathrm{~A}(\mathrm{peak})$ & 1 \\
grid voltage & $v_{g}$ & $155 \mathrm{~V}(\mathrm{l}-\mathrm{n}, \mathrm{peak})$ & 1 \\
grid frequency & $\omega$ & $2 \pi 60 \mathrm{rad} / \mathrm{s}$ & 1 \\
grid inductance & $L$ & $5 \mathrm{mH}$ & 0.12 \\
grid resistance & $R$ & $1 \Omega$ & 0.06 \\
dc-link voltage & $v_{d c}$ & $350 \mathrm{~V}$ & - \\
inverter inductance & $L_{i}$ & $5 \mathrm{mH}$ & 0.12 \\
filter capacitor & $C$ & $1.4 \mu \mathrm{F}$ & 0.008 \\
output inductance & $L_{o}$ & $2 \mathrm{mH}$ & 0.05 \\
switching frequency & $f_{s}$ & $10 \mathrm{kHz}$ & - \\
\hline
\end{tabular}

to export the relevant data from the controller to the host PC, where the data is directly plotted without additional filtering. For control, the main task provides interrupt service routines, ADC conversion, safety protections, state-machine operation mode, symmetric sequence extraction, power reference computation, current reference generation, current loop and space vector switching time computation, among others.

Regarding the control parts, it is important to notice that the sampling and switching frequency has been selected as $10 \mathrm{kHz}$. The sequence extractor has been adapted from the second order generalized integrator in [28], with a damping factor $\xi=0.7$. The current loop is being implemented as a proportional-resonant compensator tuned at the grid frequency, with gains equal to $k_{\mathrm{p}}=30 \mathrm{~V} / \mathrm{A}, k_{\text {res }}=300 \mathrm{~V} /(\mathrm{As})$. Also, the grid voltage is used as a feed-forward term [30] to improve the dynamic performance of the current loop.

The Pacific Power AC emulator allows to generate repeatable voltage sags. In fact, it can be configured to emulate short-time grid faults with time-varying dynamic profiles. This capability is used to present a test-case where the three-phase voltages evolve with time (as shown in Fig.4), and to evaluate the performance of the proposal under complex grid faults.

The rated current of the inverter is $I_{\max }=10 \mathrm{~A}$, which has been selected as the maximum current that the inverter can inject without damage, even during the voltage sag.

Between the AC emulator and the inverter, an inductiveresistive impedance has been included. The inductance value is $5 \mathrm{mH}$, and the resistance is $1 \Omega$. These values correspond to a weak grid with per unit values equal to 0.12 and 0.06 p.u. respectively. It should be noticed that these weak values has been selected so as to highlight the effects of the voltage support. However, as previously stated, the merit of the proposed control scheme does not rely on the weakness or stiffness of the grid, but in the $L / R$ ratio.

The exact value for this ratio can be difficult to obtain at run-time, since the grid impedance varies with time and the network state. To solve this inconvenient, online impedance estimators can be implemented, at the expense of increasing the controller complexity. In case that only an approximate impedance estimation is needed, other mechanisms should be implemented, as for example the knowledge of the transformers, feeders and wire material and length for the part of the 
network where the facility is connected. This second method to estimate the impedance will be less precise and the results will be less than optimal. However, the controller implementation becomes less complex.

\section{REFERENCES}

[1] E. Serban, M. Ordoñez, and C. Pondiche, "Voltage and frequency grid support strategies beyond standards," IEEE Trans. Power Electron., vol. 32, no. 1, pp. 298-309, Jan. 2017.

[2] M. Altin, O. Goksu, R. Teodorescu, P. Rodriguez, B. Bak-Jensen, and L. Helle, "Overview of recent grid codes for wind power integration," in 12th Int. Conf. on Optimization of Electrical and Electronic Equipment, May 2010, pp. 1152-1160.

[3] M. Tsili and S. Papathanassiou, "A review of grid code technical requirements for wind farms," IET Renewable Power Generation, vol. 3, no. 3, pp. 308-332, Sep. 2009.

[4] A. Vidal, F. D. Freijedo, A. G. Yepes, P. Fernandez-Comesana, J. Malvar, O. Lopez, and J. Doval-Gandoy, "Assessment and optimization of the transient response of proportional-resonant current controllers for distributed power generation systems," IEEE Trans. Ind. Electron., vol. 60 no. 4, pp. 1367-1383, Apr. 2013.

[5] S. Alepuz, S. Busquets-Monge, J. Bordonau, J. A. Martinez-Velasco, C. A. Silva, J. Pont, and J. Rodriguez, "Control strategies based on symmetrical components for grid-connected converters under voltage dips," IEEE Trans. Ind. Electron., vol. 56, no. 6, pp. 2162-2173, Jun. 2009.

[6] S. K. Chaudhary, R. Teodorescu, P. Rodriguez, P. C. Kjær, and A. Gole, "Negative sequence current control in wind power plants with VSCHVDC connection," IEEE Trans. Sustain. Energy, vol. 3, no. 3, pp. 535-544, Jul. 2012.

[7] T. Lee, S. Hu, and Y. Chan, "D-STATCOM with positive-sequence admittance and negative-sequence conductance to mitigate voltage fluctuations in high-level penetration of distributed generation systems," IEEE Trans. Ind. Electron., vol. 60, no. 4, pp. 1417-1428, Apr. 2013.

[8] C.-T. Lee, C.-W. Hsu, and P.-T. Cheng, "A low-voltage ride-through technique for grid-connected converters of distributed energy resources," IEEE Trans. Ind. Appl., vol. 47, no. 4, pp. 1821-1832, Jul. 2011.

[9] A. Moawwad, M. S. E. Moursi, and W. Xiao, "Advanced fault ridethrough management scheme for vsc-hvdc connecting offshore wind farms," IEEE Trans. Power Syst., vol. 31, no. 6, pp. 4923-4934, Nov 2016.

[10] F. Wang, J. L. Duarte, and M. A. M. Hendrix, "Pliant active and reactive power control for grid-interactive converters under unbalanced voltage dips," IEEE Trans. Power Electron., vol. 26, no. 5, pp. 1511-1521, May 2011.

[11] J. Miret, A. Camacho, M. Castilla, J. L. G. de Vicuña, and J. de la Hoz, "Reactive current injection protocol for low-power rating distributed generation sources under voltage sags," IET Power Electronics, vol. 8, no. 6, pp. 879-886, 2015.

[12] X. Du, Y. Wu, S. Gu, H. M. Tai, P. Sun, and Y. Ji, "Power oscillation analysis and control of three-phase grid-connected voltage source converters under unbalanced grid faults," IET Power Electronics, vol. 9 , no. 11 , pp. 2162-2173, 2016.

[13] A. Camacho, M. Castilla, J. Miret, R. Guzman, and A. Borrell, "Reactive power control for distributed generation power plants to comply with voltage limits during grid faults," IEEE Trans. Power Electron., vol. 29, no. 11 , pp. 6224-6234, Nov. 2014.

[14] M. Mirhosseini, J. Pou, and V. G. Agelidis, "Individual phase current control with the capability to avoid overvoltage in grid-connected photovoltaic power plants under unbalanced voltage sags," IEEE Trans. Power Electron., vol. 30, no. 10, pp. 5346-5351, Oct 2015.

[15] X. Guo, X. Zhang, B. Wang, W. Wu, and J. M. Guerrero, "Asymmetrical grid fault ride-through strategy of three-phase grid-connected inverter considering network impedance impact in low-voltage grid," IEEE Trans. Power Electron., vol. 29, no. 3, pp. 1064-1068, Mar. 2014.

[16] A. Camacho, M. Castilla, J. Miret, P. Martí, and M. Velasco, "Maximizing positive sequence voltage support in inductive-resistive grids for distributed generation inverters during voltage sags," in 42th Ann. Conf of IEEE Ind. Electron. Society, Oct 2016, pp. 2343-2348.

[17] Y. Liu, Y. Li, Y. Chi, and W. Wang, "Analysis on a large scale wind turbines cascading trip-off accident in North China," in IEEE PowerTech, Jun. 2013, pp. 1-6.
[18] A. Vidal, A. G. Yepes, F. D. Freijedo, O. López, J. Malvar, F. Baneira, and J. Doval-Gandoy, "A method for identification of the equivalent inductance and resistance in the plant model of current-controlled gridtied converters," IEEE Trans. Power Electron., vol. 30, no. 12, pp. 72457261, Dec 2015

[19] L. Asiminoaei, R. Teodorescu, F. Blaabjerg, and U. Borup, "Implementation and test of an online embedded grid impedance estimation technique for PV inverters," IEEE Trans. Ind. Electron., vol. 52, no. 4, pp. 1136 1144, Aug. 2005.

[20] A. Moallem, D. Yazdani, A. Bakhshai, and P. Jain, "Frequency domain identification of the utility grid parameters for distributed power generation systems," in 26th Ann. IEEE Applied Power Electronics Conf. and Expo. (APEC), Mar. 2011, pp. 965-969.

[21] S. Cobreces, E. J. Bueno, D. Pizarro, F. J. Rodriguez, and F. Huerta, "Grid impedance monitoring system for distributed power generation electronic interfaces," IEEE Trans. Instrum. Meas., vol. 58, no. 9, pp. 3112-3121, Sep. 2009.

[22] N. Hoffmann and F. W. Fuchs, "Minimal invasive equivalent grid impedance estimation in inductive-resistive power networks using extended Kalman filter," IEEE Trans. Power Electron., vol. 29, no. 2, pp. 631-641, Feb 2014

[23] Z. Xin, R. Zhao, P. Mattavelli, P. C. Loh, and F. Blaabjerg, "Reinvestigation of generalized integrator based filters from a first-ordersystem perspective," IEEE Access, vol. PP, no. 99, pp. 1-1, 2016.

[24] A. Camacho, M. Castilla, J. Miret, A. Borrell, and L. G. de Vicuña, "Active and reactive power strategies with peak current limitation for distributed generation inverters during unbalanced grid faults," IEEE Trans. Ind. Electron., vol. 62, no. 3, pp. 1515-1525, Mar. 2015.

[25] O. Goksu, R. Teodorescu, C. L. Bak, F. Iov, and P. C. Kjær, "Impact of wind power plant reactive current injection during asymmetrical grid faults," IET Renewable Power Generation, vol. 7, no. 5, pp. 484-492, Sep. 2013.

[26] S. Kincic, X. Wan, D. T. McGillis, A. Chandra, B.-T. Ooi, F. D. Galiana, and G. Joos, "Voltage support by distributed static VAr systems (SVS)," IEEE Trans. Power Del., vol. 20, no. 2, pp. 1541-1549, Apr. 2005.

[27] B. I. Crăciun, T. Kerekes, D. Séra, and R. Teodorescu, "Frequency support functions in large PV power plants with active power reserves," IEEE Trans. Emerg. Sel. Topics Power Electron., vol. 2, no. 4, pp. 849858, Dec 2014.

[28] F. J. Rodriguez, E. Bueno, M. Aredes, L. G. B. Rolim, F. A. S. Neves, and M. C. Cavalcanti, "Discrete-time implementation of second order generalized integrators for grid converters," in 34th Annu. Conf. of IEEE Ind. Electron., Nov. 2008, pp. 176-181.

[29] P. Rodriguez, G. Medeiros, A. Luna, M. C. Cavalcanti, and R. Teodorescu, "Safe current injection strategies for a statcom under asymmetrical grid faults," in IEEE Energy Conversion Congr. and Expo. (ECCE), Sep. 2010, pp. 3929-3935.

[30] A. Yazdani and R. Iravani, Voltage-Sourced Converters in Power Systems. New Jersey, USA: Wiley, 2010.

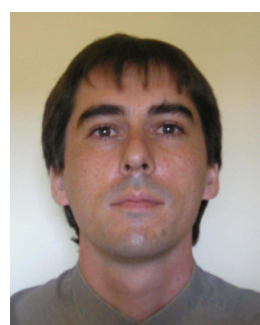

Antonio Camacho received the B.S. degree in chemical engineering, the M.S. degree in automation and industrial electronics, and the Ph.D. degree in electronic engineering, from the Technical University of Catalonia, Barcelona, Spain in 2000, 2009 and 2015 respectively.

His research interests include networked and embedded control systems, industrial informatics, and power electronics.

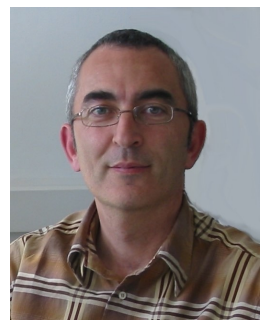

Miguel Castilla received the B.S., M.S. and Ph.D degrees in telecommunication engineering from the Technical University of Catalonia, Barcelona, Spain, in 1988, 1995, and 1998, respectively.

Since 2002, he has been an Associate Professor in the Department of Electronic Engineering, Technical University of Catalonia, where he teaches courses on analog circuits and power electronics. His research interests are in the areas of power electronics, nonlinear control, and renewable energy systems. 


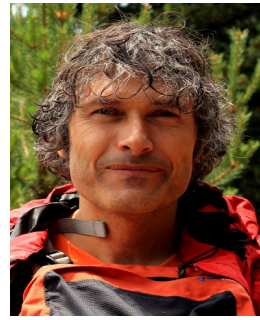

Jaume Miret (M'98) received the B.S. degree in telecommunications, M.S. degree in electronics, and Ph.D. degree in electronics from the Universitat Politecnica de Catalunya, Barcelona, Spain, in 1992, 1999, and 2005, respectively.

From 1993 to 2011, he was an Assistant Professor in the Department of Electronic Engineering, Universitat Politecnica de Catalunya, Spain. Since 2011 he has been an Associate Professor in the Universitat Politecnica de Catalunya, where he teaches courses on digital design and circuit theory. His research interests include dc-to-ac converters, active power filters, and digital control.

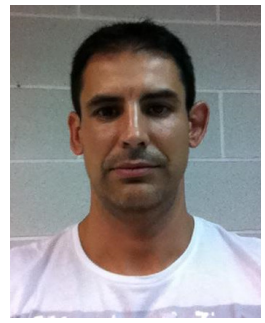

Ramon Guzman received the B.S, the M.S and the $\mathrm{Ph} . \mathrm{D}$. degrees in telecommunications engineering from the Technical University of Catalonia, Barcelona, Spain, in 1999, 2004 and 2016, respectively.

He is currently an Associate Professor with the Department of Automatic Control in the Technical University of Catalonia. His research interests include nonlinear and adaptive control for three-phase power converters.

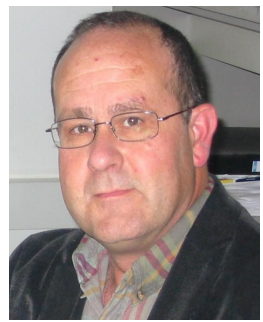

Luis García de Vicuña received the M.S. and Ph.D. degrees in Telecommunication Engineering from the Technical University of Catalonia, Barcelona, Spain, in 1980 and 1990, respectively, and the Ph.D. degree in Electrical Engineering from the Paul Sabatier University, Toulouse, France, in 1992.

$\mathrm{He}$ is currently a Full Professor with the Department of Electronic Engineering, Technical University of Catalonia, where he teaches courses on power electronics. His research interests include power electronics modeling, simulation and control, active power filtering, and high power-factor ac/dc conversion. 